Fisioter Bras 2019;20(1);84-94

http://dx.doi.org/10.33233/fb.v20i1.2732

\title{
ARTIGO ORIGINAL \\ Análise do tempo de reação simples e do paradigma oddball em estudantes antes e após participação em atividades acadêmicas: uma análise da neurociência aplicada a educação \\ Analysis of simple reaction time and the oddball paradigm in students before and after participation in academic activities: an analysis of neuroscience applied to education
}

Elivia Silva Teles ${ }^{1}$, Maria Joaquina do Carmo Neta ${ }^{1}$, Pollyana Soares Lustosa ${ }^{1}$, Kaline Rocha ${ }^{2}$, Carla Ayres $^{3}$, Pedro Ribeiro ${ }^{4}$, Bruna Velasques ${ }^{4}$, Juliana Bittencourt ${ }^{4}$, Fernando Lopes e Silva Junior $^{5,6,7,8}$, Gildário Dias ${ }^{9}$, Maurício Cagy ${ }^{10}$, Silmar Teixeira ${ }^{5,6,8}$, Rossano Fiorelli ${ }^{11}$, Marco Orsini $^{11,12}$, Eduardo Trajano ${ }^{11}$, Janaína de Moraes Silva ${ }^{12,13}$, Victor Hugo do Vale Bastos ${ }^{5,6,7}$

${ }^{1}$ Fisioterapeuta, UFPI-CMRV, Parnaíba/PI, ${ }^{2}$ Doutoranda do programa de Biotecnologia da UFPI-CMRV, Parnaíba/PI, ${ }^{3}$ Mestranda do programa de Biotecnologia da UFPI-CMRV, Parnaíba/PI, ${ }^{4}$ Laboratório de mapeamento cerebral e integração sensório-motora IPUB/UFRJ, Rio de Janeiro/RJ, ${ }^{5}$ Docente do curso de graduação da Universidade Federal do Piauí-UFPICMRV, Parnaíba/PI, ${ }^{6}$ Docente do programa de mestrado em Ciências Biomédicas da UFPICMRV, Parnaíba/PI, ' ${ }^{7}$ Laboratório de Mapeamento Cerebral e Funcionalidade UFPI-CMRVLAMCEF, Parnaíba-PI, ${ }^{8}$ Laboratório de Mapeamento e Plasticidade Cerebral UFPI-CMRVLAMPLACE, Parnaíba/PI, ${ }^{9}$ Laboratório de Neurofísica LANF-UFPI-CMRV, Parnaíba/PI, ${ }^{10}$ Programa de Engenharia Biomédica COPPE-UFRJ, ${ }^{11}$ Programa de Mestrado em Ciências Aplicadas em Saúde, Universidade de Vassouras, Vassouras/RJ, ${ }^{12}$ Pós-doutorando em Ciências Biomédicas UFPI, Parnaíba/PI, ${ }^{13}$ Docente da Universidade Estadual do PiauíUESPI/CCS, Teresina-PI, Brasil; Pós-doutoranda em Ciências Biomédicas UFPI, Parnaíba/PI

Recebido 16 de outubro de 2018; aceito 9 de janeiro de 2019.

Endereço para correspondência: Janaína de Moraes Silva, Avenida São Sebastião, 2819 São Benedito 64202-020 Parnaíba PI, E-mail: fisiojanainams@gmail.com; Elivia Silva Teles: eliviag3@hotmail.com; Maria Joaquina do Carmo Neta: ftmariajoaquina@outlook.com; Pollyana Soares Lustosa: pollylustosa7790@gmail.com; Kaline Rocha: kaline_mel@hotmail.com; Carla Ayres: carlaayres7@gmail.com; Pedro Ribeiro: ribeiropss@yahoo.com.br; Bruna Velasques: bruna_velasques@yahoo.com.br; Juliana Bittencourt: juju_bitt@yahoo.com.br; Fernando Lopes e Silva Junior: fernando.lopes@ufpi.edu.br; Gildário Dias: gildario@ufpi.edu.br; Maurício Cagy: mauricio.cagy@gmail.com; Silmar Teixeira: silmar_teixeira@yahoo.com.br; Rossano Fiorelli: fiorellirossano@hotmail.com; Marco Orsini: orsinimarco@hotmail.com; Eduardo Trajano: eduardolimatrajano@hotmail.com; Victor Hugo do Vale Bastos: victorhugobastos@ufpi.edu.br

\section{Resumo}

Introdução: O tempo de reação é uma medida que indica o tempo que uma pessoa leva para iniciar um movimento. Há situações em que o tempo de reação encontra-se alterado, comprometendo o processamento da informação, com diminuição na detecção, transmissão e processamento dos estímulos. Objetivo: Investigar o tempo de reação visual em acadêmicos antes e após atividades avaliativas, nas diversas disciplinas. Metodologia: 50 acadêmicos foram analisados antes e após atividades avaliativas por meio do tempo de reação simples e paradigma oddball. Resultados: Com relação ao tempo de reação simples, o tempo de reação visual antes das atividades avaliativas foi menor que após, em contradição com o paradigma oddball. Verificou-se que a média geral do tempo de reação simples para prova prática foi maior comparado às demais, já no paradigma oddball verificou-se que a média geral para apresentação de seminário foi maior, comparado às demais. Conclusão: Diferenças significativas no tempo de reação simples e tempo de reação segundo paradigma oddball foram encontrados entre acadêmicos antes e após atividades avaliativas. Porém no tempo de reação simples foram encontrados valores menores antes das atividades, quando comparados com após, e o contrário foi encontrado no paradigma oddball.

Palavras-chave: tempo de reação, atividades avaliativas, paradigma oddball. 


\begin{abstract}
Introduction: Reaction time is a measure of how long a person takes to start a movement. There are situations in which the reaction time is altered, compromising the information processing, with a decrease in the detection, transmission and processing of the stimuli. Objective: To investigate the time of visual reaction in academics before and after evaluative activities in different disciplines. Methodology: 50 academics were analyzed before and after evaluative activities through simple reaction time and oddball paradigm. Results: Relative to the time of simple reaction, the visual reaction time before the evaluative activities was smaller than after, in contradiction with the oddball paradigm. It was verified that the general mean of the simple reaction time for practical test was higher compared to the others, already in the oddball paradigm it was verified that the general average for seminar presentation was higher, compared to the others. Conclusion: Significant differences in the time of simple reaction and reaction time according to the oddball paradigm were found among academics before and after evaluative activities. However, in the simple reaction time smaller values were found before the activities, when compared with after, and the opposite was found in the oddball paradigm.
\end{abstract}

Key-words: reaction time, evaluative activities, oddball paradigm.

Introdução

O tempo de reação (TR) é uma medida que traduz a velocidade de processamento de uma informação, sendo assim considerado uma das medidas mais relevantes do desempenho humano [1]. O TR possui três classificações que se diferenciam, sobretudo, quanto à natureza do estímulo que pode ser visual, auditivo ou tátil; e o número de possibilidades ou alternativas do estímulo-resposta [2]. O TR simples é o intervalo de tempo referente à reação de um único sinal, já o TR de discriminação consiste no tempo decorrido entre a percepção de mais de um estímulo e o início de uma única resposta, enquanto que o TR de escolha define o intervalo de tempo entre a percepção de vários estímulos e o início de uma resposta específica para cada estímulo [3].

O TR simples representa o nível de coordenação neuromuscular nos quais os estímulos são decodificados pelo corpo por meio de diferentes processos físico-químicos e mecânicos [4]. O paradigma oddball é tradicionalmente usado, por ser uma forma de avaliação ideal de como o cérebro discrimina estímulos e probabilidade de processos. Nesse paradigma dois estímulos são apresentados aleatoriamente, com um ocorrendo em pouca frequência. Os participantes são solicitados a discriminar um estímulo alvo pré-definido (infrequente) de um não-alvo (frequente) [5]. A capacidade de memória pode assumir um papel de destaque neste processo, uma vez que é responsável pela aquisição, codificação, armazenamento e recuperação da informação, dando-nos a possibilidade de separar e organizar a informação contida nos estímulos recebidos [6]. O TR aumenta em função do número de itens da memória, havendo assim uma relação direta entre estas duas variáveis. Este pressuposto estabelece o aumento do TR, numa proporção constante, cada vez que o número de alternativas do estímulo-resposta é duplicado [7].

No final da década de 1960, uma análise por meio do eletroencefalograma (EEG) revelou que a apresentação de um estímulo produz mudanças específicas no cérebro [8]. Durante a apresentação de um estímulo, há um aumento significativo da atividade sináptica. Mudanças nos potenciais das membranas ocorrem numa fração de um segundo após o estímulo ser apresentado, em distintas regiões do cérebro [6]. Uma vez que estes potenciais sinápticos são evocados por um estímulo, elas ocorrem em um caminho sincronizado. As respostas elétricas combinadas desta população neuronal são conhecidas como potencial evocado. O potencial evocado consiste de uma série de resultados positivos e ondas negativas que podem ser nomeados numericamente ou de acordo com a sua latência. As principais ondas são: N1, P2, N2 e P3 [8].

O TR simples, além de ser um indicador de concentração e atenção é influenciável por fatores relacionados ao condicionamento físico, coordenação motora e também fatores genéticos e psicológicos [4]. Em situações em que os indivíduos são submetidos à ansiedade ou em condições de estresse, o TR pode aumentar, pois os reflexos ficam bem mais lentos e até mesmo a acuidade visual pode diminuir [9]. Nas exigências acadêmicas os alunos são submetidos constantemente a diferentes avaliações, podendo produzir um nível elevado de ansiedade. Dessa maneira, o TR desses indivíduos pode ficar alterado, causando um efeito debilitante sobre o desempenho acadêmico $[7,10]$. 
Nas situações em que o TR pode se encontrar alterado, como no caso de acadêmicos que são submetidos a meios diferentes de avaliação, poderá ocorrer um comprometimento do processamento da informação, com diminuição considerável na detecção, transmissão e processamento dos estímulos. O aumento dessa variável frente às atividades avaliativas não está bem determinado na literatura e nesse sentido, a investigação do TR visual antes e após as atividades avaliativas poderá proporcionar um incentivo para que novos estudos sejam realizados. Nesse sentido, o presente estudo teve como objetivo investigar o TR visual em acadêmicos antes e após atividades avaliativas nas diversas disciplinas.

\section{Material e métodos}

O estudo realizou-se por meio de uma pesquisa do tipo descritiva e inferencial com abordagem quantitativa e de caráter observacional. A pesquisa realizou-se na Universidade Federal do Piauí, Campus de Parnaíba. O projeto foi enviado e aprovado pelo Comitê de Ética em Pesquisas Envolvendo Seres Humanos (Parecer 735.130)

\section{Participantes}

Participaram deste estudo 50 acadêmicos, que se disponibilizaram de maneira aleatória, e que estavam regularmente matriculados. Estes foram avaliados antes e após exame teórico, prático ou apresentação de seminário. A seleção dos voluntários foi feita na própria instituição, por meio de convites realizados nas salas de aula, em todos os períodos. Os participantes foram informados sobre os procedimentos do estudo e em relação ao sigilo, e todos assinaram o termo de consentimento livre e esclarecido. Após a assinatura, os participantes foram convidados a acompanharem um pesquisador, individualmente para a sala da pesquisa.

\section{Instrumentos}

Para mensurar o TR simples e o paradigma oddball foram utilizados dois notebooks de marcas LG e Philco, Windows7 em ambos, com instalação do software Matlab e do programa Average, que calculava a amplitude em milissegundos que o participante levava para responder ao estímulo. Os computadores possuíam uma tecla de sensibilidade, que era acionada por uma carga mínima de $0,8 \mathrm{~N}$, e um monitor que projetava um estímulo visual, com distância de $50 \mathrm{~cm}$ do participante.

\section{Coleta de dados}

Os equipamentos foram posicionados em salas isoladas, às quais somente os acadêmicos e responsáveis pela pesquisa tiveram acesso, permitindo que a concentração e a atenção dos participantes ficassem focalizadas nos testes. Os sujeitos foram orientados a posicionarem-se sentados, com o antebraço apoiado sobre uma mesa e o dedo indicador sobre a tecla do computador. Além disso, foi explicado aos voluntários que dois estímulos seriam apresentados no computador de forma aleatória e que os mesmos deveriam discriminar o alvo (pouco frequente) de estímulos não alvo (frequente). Primeiramente o estimulo alvo foi representado pelo aparecimento de um círculo no meio da tela do monitor, logo após esse estimulo alvo foi representado pelo aparecimento de um quadrado e o círculo passou a ser o estímulo não alvo. Os indivíduos foram instruídos para responder o estímulo alvo pressionando o botão. Cada estudante foi submetido a um bloco de 50 estímulos para o TR simples e dois blocos de 20 estímulos com intervalo de 2 minutos entre os blocos para o paradigma oddball, em que esse estímulo apareceu na tela com duração de 0,75 segundos. Um pré-teste foi aplicado dez minutos antes objetivando a familiarização dos indivíduos com os instrumentos de medida.

\section{Análise de dados}

Os dados oriundos dos procedimentos descritos acima foram analisados no programa SPSS - Statistical Package for the Social Sciences, na versão 20.0, utilizando-se as 
ferramentas descritivas médias e desvio padrão. O teste Kolmogorov-Smirnov foi utilizado para homocedasticidade e homogeneidade da amostra e o teste Mann-Whitney foi usado para verificar as diferenças existentes entre os grupos de atividades avaliativas, e a comparação estatística antes e após estas avaliações, sendo utilizados 50 estímulos para o TR simples e 40 para o paradigma oddball. O nível de significância considerado foi de $p \leq 0.05$.

\section{Resultados}

Como resultados, diferenças estatisticamente significativas foram verificadas na comparação entre antes e após as atividades avaliativas do TR simples $[F(1,2447)=8,958$; $\left.\mathrm{p}=0,003, \eta^{2}=0,004\right]$ e entre os diferentes tipos de atividades avaliativas do TR simples $\left[F(1,2447)=18,378 ; p=0,0001, \eta^{2}=0,15\right]$. Na comparação entre antes e após a realização das atividades avaliativas do paradigma oddball $\left[F(1,1957)=0,050 ; p=0,823, \eta^{2}=0,0001\right]$ também foi encontrado diferenças, assim como na comparação entre os diferentes tipos de atividades avaliativas do paradigma oddball $\left[F(4239,000)=9,571 ; p=0,002, \eta^{2}=0,002\right]$, havendo interações para condição do paradigma oddball e não havendo para o grupo do paradigma oddball.
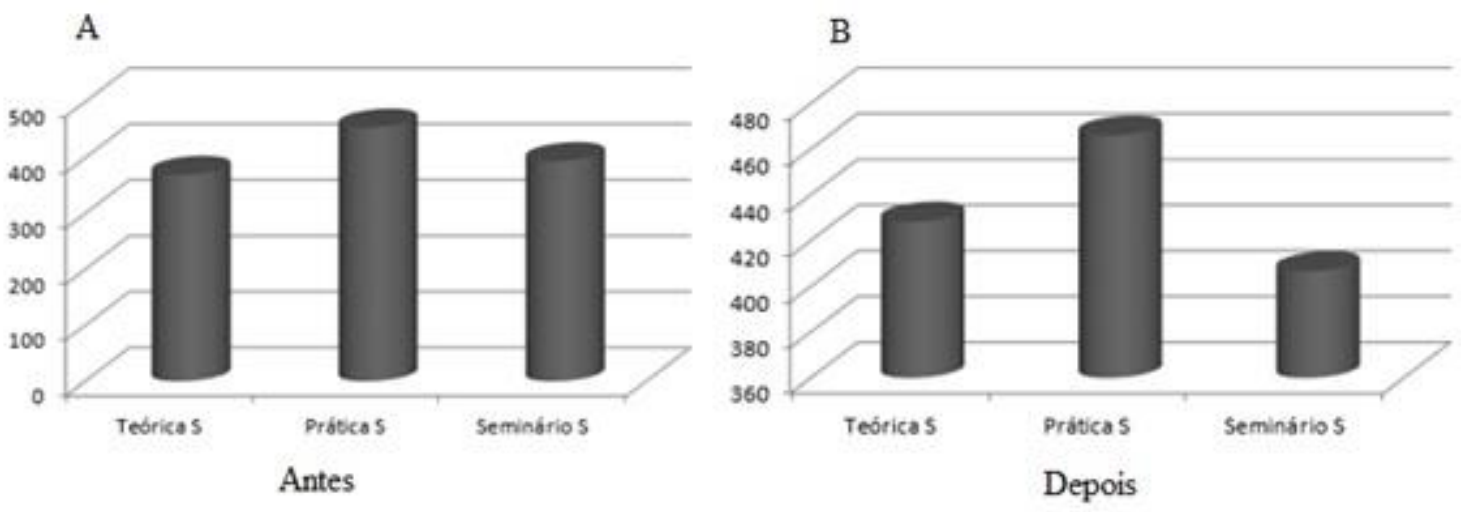

Figura 1 - Comparação estatística da média, antes (A) e após (B) a aplicação dos diferentes tipos de atividades avaliativas do tempo de reação simples.

O Figura 1A apresenta a comparação estatística da média, do tempo de TR simples antes e após das atividades avaliativas, que correspondem à média obtida dos 50 estímulos apresentados. A média geral do TR simples para prova prática $(453,87 \mathrm{~ms})$ foi maior quando comparado com a média de apresentação de seminário $(393,84 \mathrm{~ms})$ e prova teórica $(369,41$ $\mathrm{ms}$ ), sem diferenças significativas. O Figura 1B apresenta a comparação estatística da média do TR simples após as atividades avaliativas, que correspondem à média obtida dos 50 estímulos apresentados. A média geral do TR simples para prova prática $(465,92 \mathrm{~ms})$ foi maior quando comparado com a média da prova teórica $(428,27 \mathrm{~ms})$ e apresentação de seminário (406,64 ms), sem diferenças significativas. 
A

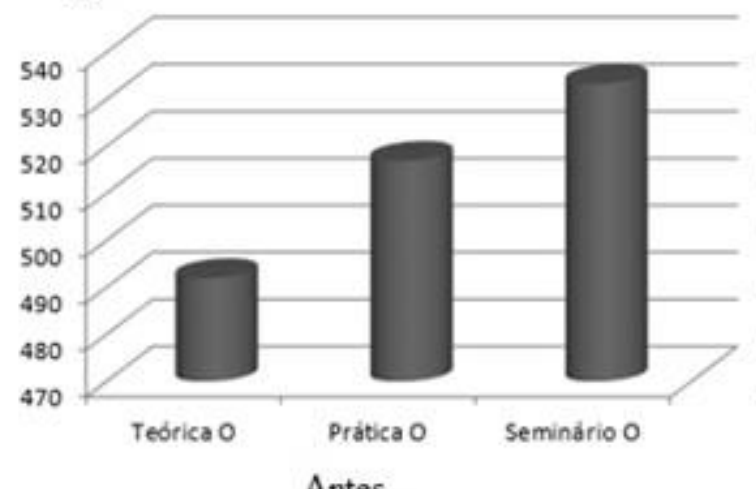

B

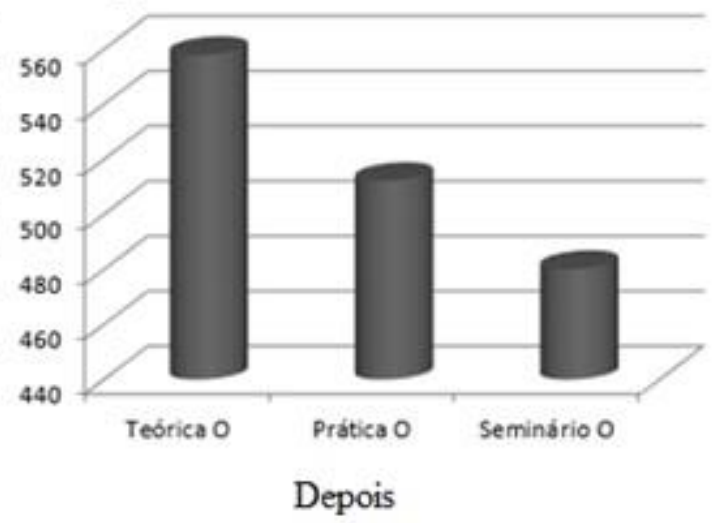

Figura 2 - Comparação estatística da média antes $(A)$ e após (B) da aplicação dos diferentes tipos de atividades avaliativas do paradigma oddball.

A Figura $2 \mathrm{~A}$ apresenta a comparação estatística da média do paradigma oddball antes das atividades avaliativas, que correspondem à média obtida dos 40 estímulos apresentados. $\mathrm{A}$ média geral do paradigma oddball para apresentação de seminário $(533,35 \mathrm{~ms})$ foi maior quando comparado com a média da prova prática $(516,95 \mathrm{~ms})$ e prova teórica $(491,99 \mathrm{~ms})$, sem diferenças significativas. $\mathrm{O}$ Figura $2 \mathrm{~B}$ apresenta a comparação estatística da média, erro padrão da média e valor de $\mathrm{N}$ do paradigma oddball após as atividades avaliativas, que correspondem à média obtida dos 40 estímulos apresentados. A média geral do paradigma oddball para prova teórica $(557,85 \mathrm{~ms})$ foi maior quando comparado com a média da prova prática $(512,14 \mathrm{~ms})$ e apresentação de seminário $(480,13 \mathrm{~ms})$, sem diferenças significativas.

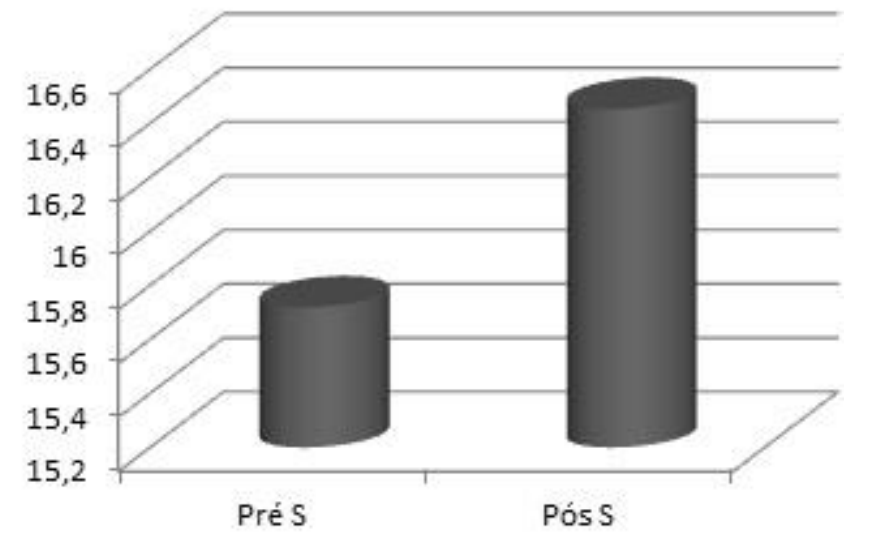

Figura 3 - Tempo de reação simples antes e após prova teórica.

A Figura 3 apresenta o TR simples antes e após a atividade avaliativa do tipo teórica, que correspondem aos valores do erro padrão. O TR simples antes da prova teórica foi menor $(15,718)$ quando comparado após a realização da mesma $(16,453)$, com diferenças significativas. 


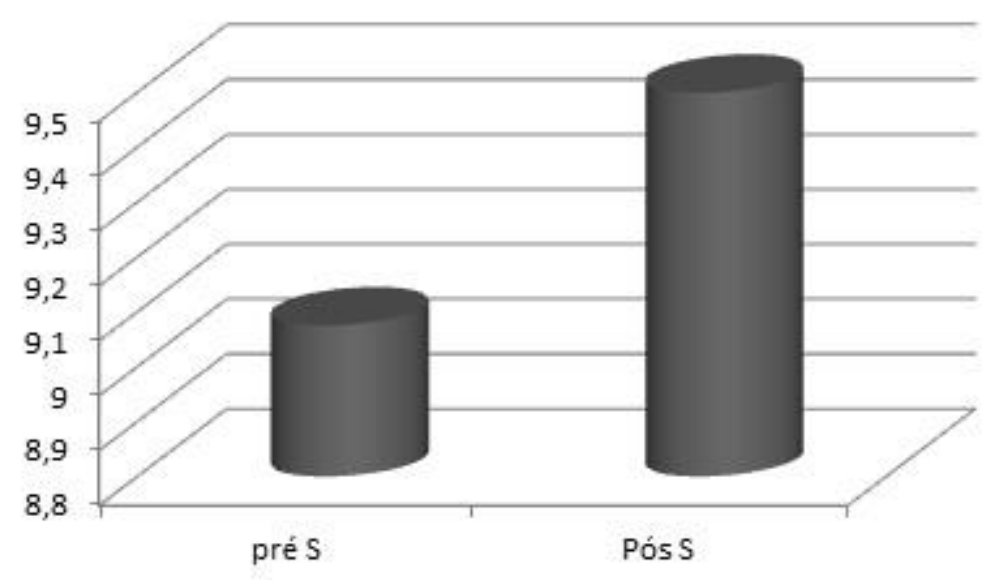

Figura 4 - Tempo de reação simples antes e após prova prática.

O Figura 4 apresenta o TR simples antes e após a atividade avaliativa do tipo prática, que correspondem aos valores de erro padrão. O TR simples antes da prova prática foi menor $(9,075)$ quando comparado após a realização da mesma $(9,499)$, com diferenças significativas.

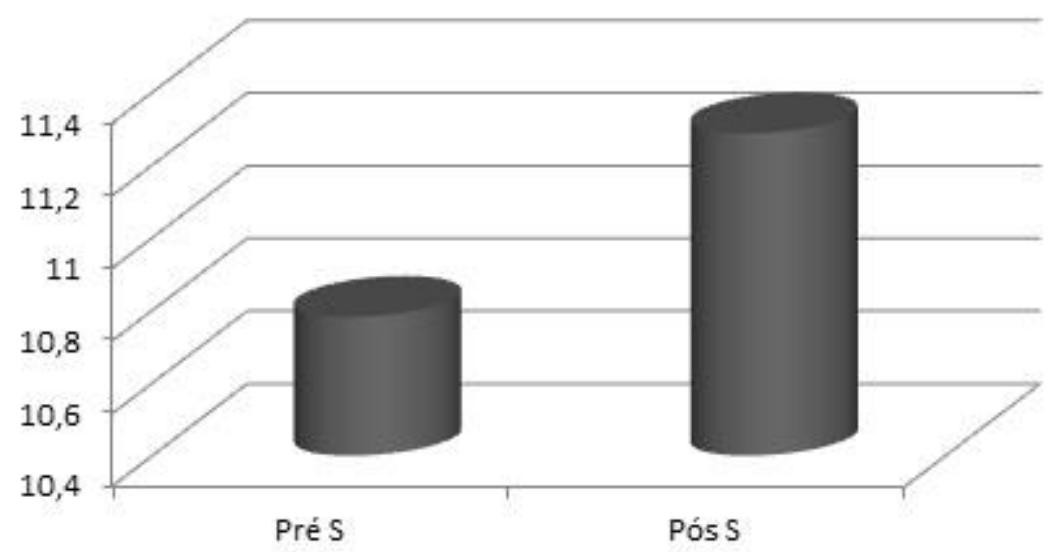

Figura 5 - Tempo de reação simples antes e após apresentação de seminário.

O Figura 5 apresenta o TR simples antes e após a atividade avaliativa do tipo seminário, que correspondem aos valores de erro padrão. O TR simples antes da apresentação de seminário foi menor $(10,782)$ quando comparado após a realização da mesma $(11,287)$, com diferenças significativas.

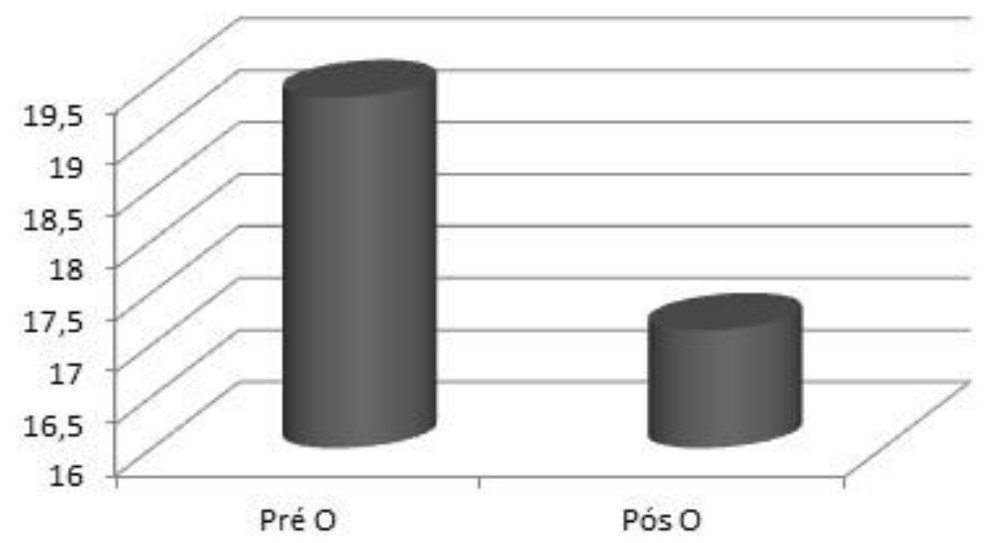

Figura 6 - Paradigma oddball antes e após prova teórica. 
A Figura 6 apresenta a comparação estatística do paradigma oddball antes e após a atividade avaliativa do tipo teórica, que correspondem aos valores de erro padrão. O TR segundo o paradigma oddball antes da prova teórica foi maior $(19,378)$ quando comparado após a realização da mesma $(17,133)$, com diferenças significativas.

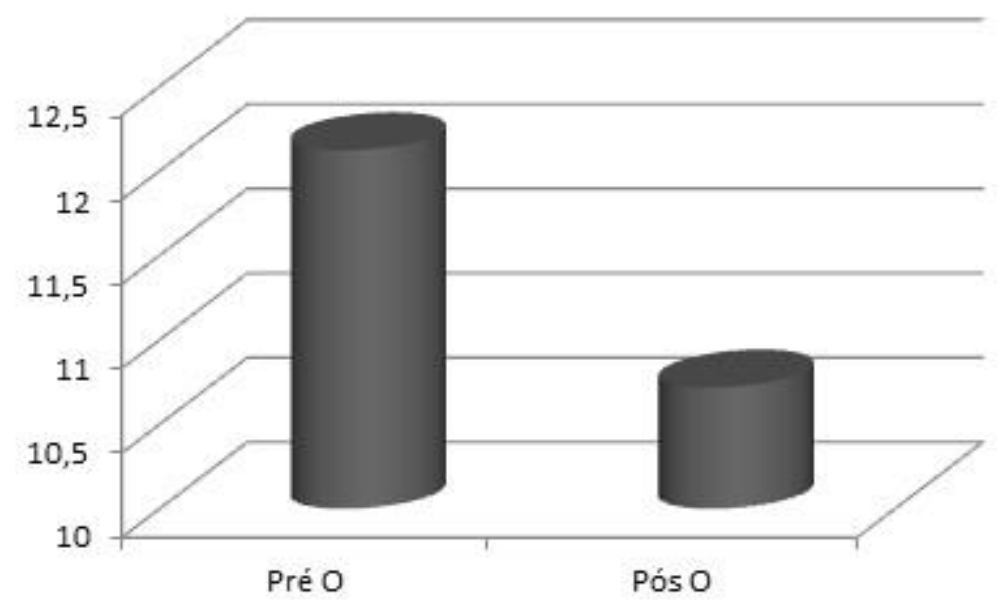

Figura 7 - Paradigma oddball antes e após prova prática.

A Figura 7 apresenta a comparação do paradigma oddball antes e após a atividade avaliativa do tipo prática, que correspondem aos valores de erro padrão. O TR segundo 0 paradigma oddball antes da prova prática foi maior $(12,122)$ quando comparado após a realização da mesma $(10,718)$, com diferenças significativas.

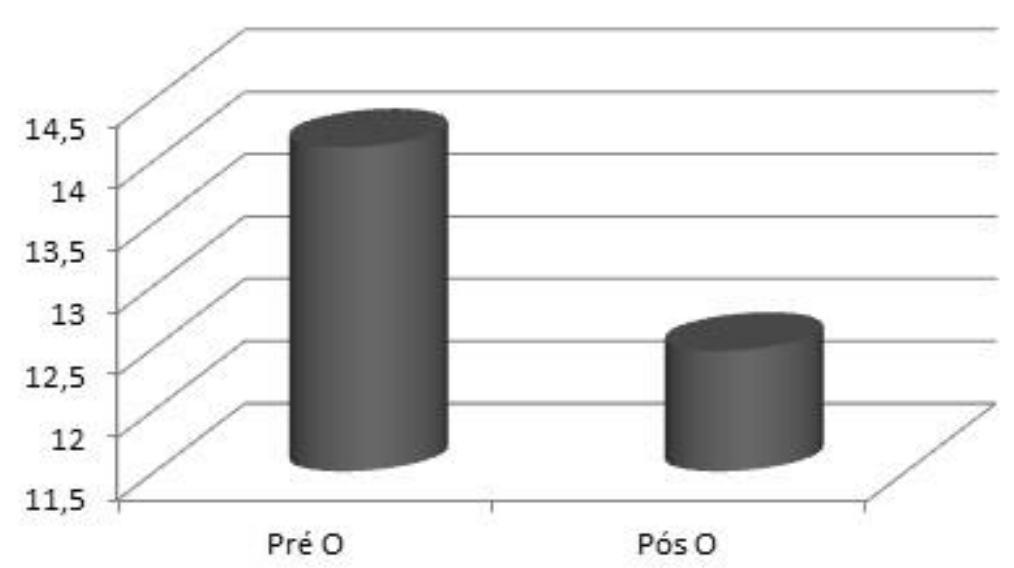

Figura 8 - Paradigma oddball antes e após apresentação de seminário.

A Figura 8 apresenta a comparação estatística do paradigma oddball antes e após a atividade avaliativa do tipo seminário, que correspondem aos valores de erro padrão. O TR segundo o paradigma oddball antes da apresentação de seminário foi maior (14,1) quando comparado após a realização da mesma $(12,466)$, com diferenças significativas.

\section{Discussão}

O TR pode ser definido como o intervalo de tempo decorrido desde um estímulo - que pode ser auditivo ou visual - até o início de uma resposta, sendo utilizado por pesquisadores como uma medida do desempenho sensório-motor [11-13]. Atrasos na reação ao estímulo podem ser de significância crítica na determinação do sucesso em habilidades que requerem respostas rápidas, sendo crucial para acadêmicos, que são constantemente submetidos a 
meios avaliativos, onde precisam associar as questões os quais são interrogados com suas referidas respostas em um curto e determinado tempo [14].

O TR varia de acordo com alguns fatores, dentre os principais, a modalidade sensorial do estímulo e a complexidade da resposta a ser executada. Responder a estímulos acústicos, óticos e táteis implica em TRs diferentes [15]. A amplitude em milissegundos para o TR é de 130 a 170 (acústico - sinal sonoro), 200 a 250 (visual - luz), 150 a 160 (tátil simples - toque) e 510 a 530 (tátil complexo - rotação do corpo) [16], sendo que esses valores do TR é um dos principais indicadores da limitação neuromotora para processar um estímulo do ambiente externo [15].

Em situações nas quais dois estímulos são apresentados inesperadamente muito próximos, o encéfalo capta o primeiro estímulo e começa a selecionar e gerar uma resposta para ele. O segundo estímulo é então apresentado, e ocorre uma interferência com o segundo par de estímulo e resposta. Esse atraso do TR é mais longo quando o estímulo e resposta é muito curto (aproximadamente 50 a $60 \mathrm{~ms}$ ), mas se o estímulo for menos de $40 \mathrm{~ms}$, o encéfalo responde aos dois estímulos simultaneamente, como se eles fossem um. Isso é denominado período psicológico refratário [12]. O TR de escolha é mais demorado porque a latência do processamento mental é mais longa, ocorrendo a Lei de Hick [17]. A Lei de Hick o TR aumenta logaritmicamente à medida que aumenta a quantidade de estímulo e resposta. Portanto, dois ou três estímulos de ataque quase ao mesmo tempo retardam o TR do defensor por causa do número de opções de escolha para esse atleta efetuar a resposta. Durante a comparação entre os dois meios em que foi avaliado o TR neste estudo, encontraram-se valores diferentes para o TR simples - em que se utilizou apenas um estímulo e para o paradigma oddball - em que foi utilizado dois estímulos diferentes, tanto antes como após as atividades avaliativas.

Uma resposta rápida está dependente do modo como a informação possa estar armazenada e organizada no sistema de memória, uma vez que facilita o processo de recuperação [18]. Esta situação pressupõe que a memória poderá ser relevante apenas em tarefas que impliquem escolhas - como é o caso das provas de TR de escolha utilizadas no presente estudo - em que é necessário evocar/recuperar a informação armazenada na memória a um curto e longo prazo [19]. Assim como mostrou os resultados deste estudo, onde o TR foi maior antes das atividades avaliativas quando comparado após, apenas quando foi necessário distinguir dois estímulos - paradigma oddball. Este fato pode constituir a explicação para as diferenças encontradas em termos médios, à medida que a complexidade da tarefa aumenta. No entanto, é necessário, em estudos futuros, aumentar a complexidade da tarefa para que se possa clarificar esta questão [16].

A resposta antecipatória significa uma disponibilidade do sistema nervoso em termos de processamento, portanto ela é adquirida com a prática, enquanto que a resposta adiantada é característica do iniciante, ou seja, resposta errada. Em um estudo foi analisado que o efeito do TR curto (TRC) ou o TR longo (TRL) no resultado de performance numa tarefa de timing antecipatório foi diluído durante o processo de aprendizagem, ou seja, após um período de prática, o grupo TRL, que tinha um desempenho inferior, se equiparou ao desempenho do grupo TRC [20].

Atrasos na reação podem ser de relevância na determinação do sucesso em habilidades rápidas, no caso de jogadores de futebol em situações como antecipar-se ao adversário em uma roubada de bola ou interceptar um chute a gol, assim como no caso de estudantes, onde precisam dar uma resposta rápida a situações onde são constantemente submetidos, como avaliações orais [12]. No presente estudo, foram encontrados valores maiores no TR em atividades que exigiam exposições orais (prova prática e apresentação de seminário) quando comparado a exposições não orais (prova teórica) quando comparado a relação entre os grupos. Em um estudo foi comparado o TR visual de estudantes universitários com estudantes de segundo grau, nos quais não foram encontradas diferenças, pois indivíduos com níveis de habilidade similares tendem a possuir valores para TR dentro de intervalos de tempo específicos [21]. No presente estudo, ao ser avaliado o TR simples, observou-se valores maiores do TR após as atividades avaliativas quando comparado antes destas. Isso pode ser pelo fato que os acadêmicos teriam pressa para o término da pesquisa, pelo medo de perderem o horário de suas avaliações.

Apesar dessa diferença entre os valores encontrados do TR simples com o paradigma oddball, esse resultado não deixa de ser relevante, uma vez que, em condições normais, quando a quantidade de informação aumenta e o sujeito tenta manter uma resposta rápida, o número de erros também aumenta [22,23]. Esta situação está associada ao compromisso velocidade-exatidão assumida pelo sujeito em tarefas desta natureza, existindo duas razões 
principais para que tal aconteça: a primeira relaciona-se com a antecipação da resposta e a segunda com uma resposta sem preparação [24].

$\mathrm{O}$ ambiente acadêmico tem um impacto diferente em cada estudante, variando de acordo com as vivências de cada um deles [25-27]. No entanto, e independentemente da constituição de cada aluno, algumas variáveis do contexto acadêmico são suficientemente importantes por si próprias, já que, além das mudanças próprias de ensino, os alunos se deparam com as incertezas naturais da escolha profissional [28]. Alguns alunos sentem ansiedade e passam por situações de estresse apenas em disciplinas que consideram relativamente difíceis, como as voltadas para a clínica, não dando relevância as disciplinas básicas. Este fato também pode explicar o porquê dos resultados encontrados neste estudo.

Estudos com equipes adultas apontam uma tendência de um maior desenvolvimento da velocidade de resposta em atletas que executam múltiplas funções dentro da equipe, indicando assim que o TR pode ser considerado um dos principais parâmetros que indique a velocidade de processamento de informações de um indivíduo [29]. No esporte, o TR representa o tempo que o atleta necessita para tomar decisões e iniciar as ações pertinentes a situação apresentada. O TR comumente é utilizado como um dos referenciais para análise do desempenho dos atletas que praticam o desporto [30-32]. Assim como no esporte, o TR também pode ser utilizado para analisar o desempenho dos acadêmicos que são submetidos constantemente a situações que necessitam de uma resposta rápida, como nos casos da realização das atividades avaliativas, principalmente aquelas que precisam de exposição oral.

O tempo que um indivíduo leva para responder a um estímulo visual é de $250 \mathrm{~ms}$ $[11,33]$. No presente estudo, os valores do TR foram consideravelmente maiores do que aqueles sugeridos pelo autor, tanto antes como após às atividades avaliativas. Em outros estudos conduzidos com atletas de diferentes modalidades, os valores do TR são menores do que aqueles propostos por esse autor, diferentes dos valores encontrados neste estudo, o que pode ser explicado pelo fato dos sujeitos serem diferentes. Em outro estudo, foram encontrados médias de $192 \mathrm{~ms}$ para atletas de atletismo, $201 \mathrm{~ms}$ para jogadores de basquetebol, $200 \mathrm{~ms}$ para atletas de ginástica artística, $221 \mathrm{~ms}$ para nadadores e $225 \mathrm{~ms}$ para pugilistas, todos do sexo masculino [34]. Neste estudo, foram encontrados valores diferentes. Deste modo, ainda persiste o debate questionando se o TR visual é influenciado ou não nos meios avaliativos em ambiente acadêmico.

Os valores encontrados para o TR simples foram mais altos do que para o TR segundo o paradigma oddball, o que parece coerente com as solicitações impostas pelos dois testes. $O$ teste para o TR simples tem um maior número de estímulos-alvo a serem processados pelo acadêmico, tendendo ultrapassar o limiar de capacidade de memória de trabalho, elevando o TR Visual [35]. Além disso, observou-se que a média geral do TR simples para prova prática $(465,92 \mathrm{~ms})$ foi maior comparado às demais, já no paradigma oddball verificou-se que a média geral para apresentação de seminário $(533,35 \mathrm{~ms})$ foi maior comparado às demais. $\mathrm{O}$ que mostra que o TR se torna maior em atividades que exigem uma apresentação oral por parte do aluno.

Em um estudo feito com 53 jovens jogadores de futebol com idade entre 8 a 13 anos, foi analisado o TR utilizando uma televisão, que iniciava com tela escura e depois ficava escuro um círculo e imediatamente o atleta chutava a bola. Um sensor detectava o momento da movimentação da bola que era transferido os dados para um computador. Os jogadores de 12 e 13 anos tiveram melhor TR $(0,387 \pm 0,034 \mathrm{~ms})$ do que os atletas de 10 e 11 anos $(0,406 \pm 0,046$ $\mathrm{ms})$ e foram superiores do que os futebolistas com 8 e 9 anos $(0,412 \pm 0,062 \mathrm{~ms})$ [36]. Neste estudo, não foram questionados a idade dos acadêmicos e o período em que estavam cursando, porém, alunos dos primeiros períodos foram os que mais participaram deste estudo. Tal dado exemplifica a necessidade de um controle mais acurado sobre a variável idade, procurando maiores evidências em estudos futuros.

Sendo pesquisada a relação entre idade e atenção foi realizada uma pesquisa com a participação de três grupos com 14 sujeitos cada, cujas idades variaram de 12 a 15 anos, de 24 a 38 anos e de 60 a 75 anos, respectivamente, onde a tarefa consistiu em localizar um alvo previamente definido após uma dica visual e nos seus resultados foram verificados que os idosos apresentaram, sistematicamente, um desempenho pior do que os adultos jovens e os adolescentes, porém não houve diferenças entre esses dois últimos grupos sugerindo assim que o pior desempenho verificado nos idosos poderia ser resultado de deficiências orgânicas decorrentes do envelhecimento [37]. O estudo anterior assemelha-se com este quanto a diferença de idade, demonstrando que indivíduos com menor idade possuem TR maior que os com maior idade. No presente estudo, participaram mais acadêmicos dos primeiros períodos, 0 
que pode haver relação com os resultados encontrados, visto que estes alunos ainda estão em processo inicial de formação acadêmica, e a transição na vida acadêmica dos estudantes no início de seus estudos universitários pode gerar um aumento de responsabilidade, ansiedade e competitividade [25].

\section{Conclusão}

De acordo com os resultados encontrados no presente estudo, pode-se concluir que houve uma diferença significativa no TR simples e paradigma oddball entre acadêmicos antes e após atividades avaliativas. Porém houve uma controvérsia com relação aos dois meios utilizados para avaliar o TR, no simples foram encontrados valores menores antes das atividades quando comparados com após e o contrário foi encontrado no paradigma oddball. Já com relação à comparação entre os grupos, destacou-se valores maiores no TR em atividades que exigiam exposições orais (prova prática e apresentação de seminário) quando comparado a exposições não orais (prova teórica).

Referências

1. Souza AP et al. Medidas de tempo de reação simples em jogadores profissionais de voleibol. Revista Digital - Buenos Aires, 2006;10(93).

2. Maciel RN, Morales AP, Ferreira LA. Comparação do tempo de reação simples e de escolha entre atletas de voleibol. Inter Science Place 2012; 22(1):131-39. https://doi.org/10.6020/1679-9844/2207

3. Aguiar RA et al. Efeito da ingestão de cafeína em diferentes tarefas de tempo de reação. Rev Bras Ciênc Esporte 2012;34(2):465-76. https://doi.org/10.1590/s010132892012000200015

4. Vaghetti CAO, Roesler $\mathrm{H}$, Andrade A. Tempo de reação simples auditivo e visual em surfistas com diferentes níveis de habilidade: comparação entre atletas profissionais, amadores e praticantes. Rev Bras Med Esporte 2007;13(2):81-85 https://doi.org/10.1590/s1517-86922007000200003

5. Portella C, Machado S, Paes F, Cagy M, Sack AT, Sandoval-Carrillo A et al. Differences in early and late stages of information processing between slow versus fast participants. Int Arch Med 2014;7(1):49. https://doi.org/10.1186/1755-7682-7-49

6. Huettel AS, McCarthy G. What is odd in the oddball task? Prefrontal cortex is activated by dynamic changes in response strategy. Neuropsychologia 2004;42:379-86. https://doi.org/10.1016/i.neuropsychologia.2003.07.009

7. Cid L, Morgado S, Vitorino A. Memória e tempo de reação de estudantes do ensino superior da área do desporto. Rev Psicol 2012;1(4):411-8.

8. Veiga H, Deslandes A, Cagy M, McDowell K, Pompeu F, Piedade R, Ribeiro P. Visual event-related potential (P300). Arq Neuropsiquiatr 2004;62(3A):575-81. https://doi.org/10.1590/s0004-282x2004000400002

9. Rueda FJM. Desempenho no teste de atenção dividida como resultado da idade das pessoas. Estud Psicol 2011;28(2):251-9. https://doi.org/10.1590/s0103166x2011000200012

10. Duchesne M, Mattos $P$. Normatização de um teste computadorizado de atenção visual. Arq Neuropsiquiatr 1997;55(1):62-9 https://doi.org/10.1590/s0004-282x1997000100010

11. Magill RA. Aprendizagem motora: conceitos e aplicações. São Paulo: Edgard Blücher; 2000.

12. Schmidt RA, Wrisberg CA. Aprendizagem e performance motora. Porto Alegre: Artmed; 2001.

13. Lefthus GK. Sensorimotor performance and limb preference. Percept Mot Skills 1981;52:683-93. https://doi.org/10.2466/pms.1981.52.3.683

14. Williams AM. Perceptual skill in soccer: Implications for talent identification and development. J Sports Sci 2000;18:737-50. https://doi.org/10.1080/02640410050120113

15. Miyamoto RJ, Meira Jr CM. Tempo de reação e tempo das provas de 50 e 100 metros rasos do atletismo em federados e não federados. Revista Portuguesa de Ciências do Desporto 2004;4(3):42-8. https://doi.org/10.5628/rpcd.04.03.42

16. Schmidt R. Motor control and learning. A behavioural emphasis. Illinois: Human Kinetics; 1988. 
17. Tani G. A arte e a ciência da finta. In: Garganta J, Oliveira J, Murad M eds. Futebol de muitas cores e sabores. Porto: Campo das Letras; 2004. p.239-46.

18. Singer R. El aprendizaje de las aciones motrices en el deporte. Barcelona: Hispano Europea; 1986.

19. Baddeley A. Essentials of human memory. Hove: Psychology Press; 1999.

20. Fleury M, Bard C. Age, stimulus velocity and task complexity as determiners of coincident timing behavior. Journal of Human Movement Studies 1985;11:305-17.

21. Kida N, Oda S, Matsumurab M. Intensive baseball practice improves the go/nogo reaction time, but not the simple reaction time. Brain Res Cogn Brain Res 2005;22:25764. https://doi.org/10.1016/i.cogbrainres.2004.09.003

22. Alves J. Processamento da informação e inteligência. Lisboa: FMH; 1995.

23. Wessel JR, Aron AR. It's not too late: The onset of the frontocentral P3 indexes successful response inhibition in the stop-signal paradigma. Psychophysiology 2015;52:472-80. https://doi.org/10.1111/psyp.12374

24. Proteau L, Girouard Y. La prise de décision rapide en situation de choix dichotomique: Une approche intégrée qui tient compte de l'amorce et de l'exécution de la réponse. Revue Canadienne de Psychologie 1987;41(4):442-73. https://doi.org/10.1037/h0084250

25. Almeida LS, Soares APC Ferreira JAG. Adaptação, rendimento e desenvolvimento dos estudantes no ensino superior: construção do questionário de vivências académicas. Methodus 2001:3-20.

26. Pololi LH, Evans AT, Reboli AC, Coplit LD, Stuber ML, Vasiliou V et al. Assessing the learning environment for medical students: an evaluation of a novel survey instrument in four medical schools. Acad Psychiatry 2017;41(3):354-9. https://doi.org/10.1007/s40596-016-0620-1

27. Wisneski JE, Ozogul G, Bichelmeyer BA. Investigating the impact of learning environments on undergraduate students' academic performance in a prerequisite and post-requisite course sequence. Internet High Educ 2017;32:1-10. https://doi.org/10.1016/i.iheduc.2016.08.003

28. Calais SL, Andrade LMB, Lipp MEN. Diferenças de sexo e escolaridade na manifestação de stress em adultos jovens. Psicol Reflex Crít 2003;16(2):257-63. https://doi.org/10.1590/s0102-79722003000200005

29. Rocha MA, Barbanti VJ. Análise das ações de saltos de ataque, bloqueio e levantamento no voleibol feminino. Revista Brasileira de Cineantropometria e Desenvolvimento Humano 2007;9(3):284-90.

30. Maciel RN, Morales AP, Barcelos JL, Nunes WJ, Azevedo MMA, Silva VF. Relação entre tempo de reação e função específica em jogadores de voleibol. Fitness \& Performance Journal 2009; 8(6).

31. Wilkerson GB, Simpson KA, Clark RA. Assessment and training of visuomotor reaction time for football injury prevention. J Sport Rehabil 2016;26(1):26-34. https://doi.org/10.1123/jsr.2015-0068

32. Hukkanen $\mathrm{E}$, Häkkinen $\mathrm{K}$. Effects of sparring load on reaction speed and punch force during the pre-competition and competition periods in boxing. J Strength Cond Res 2017;31(6):1563-8. https://doi.org/10.1519/jsc.0000000000001885

33. Jain A, Bansal R, Kumar A, Singh KD. A comparative study of visual and auditory reaction times on the basis of gender and physical activity levels of medical first year students. Int J Appl Basic Med Res 2015;5(2):124-7. https://doi.org/10.4103/2229516x.157168

34. Soares J, Osorno LAN, Palafox GHM. Estudo comparativo do tempo de reação visuomanual simples em praticantes de esporte. Kinesis, Santa Maria 1987;3 (1):77-85.

35. Vasconcelos M, Albuquerque PB. Dissociações entre tarefas de memória: Evidência para uma distinção entre as memórias implícita e explícita. Anál Psicol 2006;24(4):51923. https://doi.org/10.14417/ap.550

36. Montés-Micó R, Bueno I, Candel J, Pons A. Eye-hand and eye-foot visual reaction times of young soccer players. Optom 2000;71(12):775-80.

37. Pesce RP, Assis SG, Avanci JQ, Santos NC, Malaquias JV, Carvalhaes R. Adaptação transcultural, confiabilidade e validade da escala de resiliência. Cad Saúde Pública 2005;21(2):436-48. https://doi.org/10.1590/s0102-311x2005000200010 